\title{
The energy based devices for vaginal "rejuvenation," urinary incontinence, vaginal cosmetic procedures, and other vulvo-vaginal disorders: An international multidisciplinary expert panel opinion
}

\author{
Giuseppe Alessandro Digesu ${ }^{1}$ (D) | Visha Tailor $^{1}$ | Mario Preti ${ }^{2}$

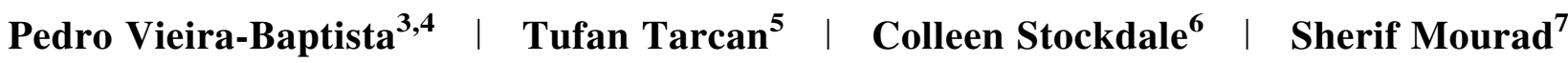

${ }^{1}$ Imperial College Healthcare, Department of Urogynaecology, London, UK

${ }^{2}$ Department of Obstetrics and Gynecology, University of Torino, Torino, Italy

${ }^{3}$ Hospital Lusíadas Porto, Porto, Portugal

${ }^{4}$ Lower Genital Tract Unit, Centro Hospitalar de São João, Porto, Portugal

${ }^{5}$ Marmara University School of Medicine, Department of Urology, Istanbul, Turkey

${ }^{6}$ Department of Obstetrics and Gynecology, University of Iowa, Iowa City, Iowa

${ }^{7}$ Ain Shams University, Department of Urology, Cairo, Egypt

\section{Correspondence}

Giuseppe Alessandro Digesu, St Marys Hospital, Imperial College Healthcare, Department of Urogynaecology, 4th floor, Mary Stanford Wing, Winsland Street, London, United Kingdom, W2 1NY.

Email: a.digesu@imperial.ac.uk

\begin{abstract}
Aims: Energy-based devices using radiofrequency and laser technologies have gained popularity as therapies for vaginal atrophy, urinary incontinence, and vaginal prolapse. They have been promoted by cosmetic and aesthetic industries for vaginal "laxity" and vaginal "rejuvenation," both of which are undefined conditions and terms. This article aims to review the current available literature and its quality on this emerging technology.
\end{abstract}

Methods: An international panel of gynaecologists, urogynaecologists, and urologists undertook a review of the available published literature, identifying articles, guidance, and society statements on the use vaginal energy-based devices. Results: There is currently no formal guidance for the use of vaginal energy based therapies. No randomized controlled trials have been published. No comparative studies to existing treatment has been carried out. Studies suggest that vaginal laser can be used in the treatment of vaginal prolapse or "vaginal laxity" and stress urinary incontinence with no quality evidence supporting the use of the therapy for vaginal atrophy or lichen sclerosis.

Conclusions: This international group propose that whilst there remains a paucity of good quality data describing the safety, benefits, and appropriate use of vaginal radiofrequency or laser treatments in gynaecology and urogynaecology, a consensus best practice document by an established scientific community needs to be developed.

\section{KEYW ORDS}

vaginal laser, vaginal laxity, vaginal rejuvenation, vaginal therapy

\section{1 | INTRODUCTION}

Energy-based devices and laser technology have FDA approval for incision, excision, ablation, vaporization, coagulation, and hemostasis of various soft tissues. The technology is used by both medical and surgical specialities including dermatology, plastic surgery, podiatry, otolaryngology, gynaecology, neurosurgery, orthopaedics, thoracic surgery, dental and oral surgery, and genitourinary surgery." nating" non-surgical procedures utilizing these same radiofrequency and laser technologies are growing in popularity.

Vaginal "rejuvenation" is a descriptive non-scientific term used by the medical device and cosmetic industries for nonsurgical vaginal cosmetic procedures or therapies. Its use is 
promoted to treat symptoms related to menopause, urinary incontinence, or to improve sexual function. ${ }^{3}$ The procedure has alternatively been labeled as cosmetic gynaecology, "revirgination" surgery, female genital plastic surgery, designer vaginal surgery, and G-spot amplification. These non-specific names have been used interchangeably with no clear standardized or scientific definition or procedure protocol. They also lack clarity and are not recognized by influential scientific communities or the Food and Drug Administration (FDA), who to date, have not cleared or approved energy based devices for any of the aforementioned vaginal procedures. ${ }^{3-8}$

Vaginal rejuvenation is advertised as a treatment for a diverse range of vaginal changes secondary to estrogen deficiency and aging (eg, vaginal atrophy and dryness), childbirth (eg, vaginal laxity or prolapse symptoms) as well as symptoms of vaginal itching, dyspareunia, pain during urination, and decreased sexual sensation. The therapy commonly utilizes radiofrequency or laser energy with a regime of repeated treatments twice or thrice every 4-6 weeks. The average cost of each treatment ranges between $£ 400$ and $£ 1000$. The therapy has a broad range of treatment aim for example to "tighten" the vagina, to treat vaginal atrophy, to eliminate dryness and/or itching, to stop incontinence, to treat pain during sexual intercourse or during micturition, to improve the sexual sensation.

Concerns regarding the use of laser and radiofrequency are not new and have previously been raised by several authors ${ }^{9,10}$ and international societies, including the American College of Obstetricians and Gynecologists (ACOG), ${ }^{11}$ the Society of Obstetricians and Gynecologists of Canada, ${ }^{12}$ the International Society for the Study of Vulvovaginal Disease (ISSVD) ${ }^{13}$ and the North American Menopause Society (NAMS). Despite recent publications alluding to subjective improvements in vaginal itching, dryness, sexual function, dyspareunia, and vaginal laxity, many studies have demonstrated contradictory and hard to explain results. ${ }^{8,14}$

Many studies reporting vaginal laser outcomes are industry sponsored, retrospective, non-randomized, short-term, lacking a control group, and utilize non-standardized or validated outcome measures with risks of significant bias. Omissions of reported complications is of concern and should not be ignored. Appropriately the FDA have therefore cautioned women considering vaginal "rejuvenation" procedures. In a supporting Safety Communication issued on 30th July 2018, the FDA state that "these products have serious risks and do not have adequate evidence to support their use for these purposes. We are deeply concerned women are being harmed."

The FDA urges women to heed their warnings, they have emphasized that women who are considering energy based vaginal procedures utilising radiofrequency and/or laser to treat the described symptoms above can lead to serious adverse events. Potential complications of vaginal burns, scarring, dryness, infection, altered sensation, dyspareunia, adhesions, scarring, recurring pain, or vaginal stenosis over time $^{2}$ may occur. These could cause sexual dysfunction or worsening quality of life as a result of radiofrequency and laser vaginal procedures for vaginal "rejuvenation." In July 2018, the FDA Commissioner Scott Gottlieb, M.D. reported on these adverse effects following an FDA review; "... the full extent of the risks is unknown. But these reports indicate these procedures can cause serious harm." ${ }^{15}$ The FDA informed the public of alarming results following vaginal laser and energy-based therapies, these are available for public review on the FDA's Adverse Event Report Databases.

Given this pertinent advice from the FDA, it is indisputable that despite promising availability of short-term data, more robust, sham-controlled, and knowledge of longer-term outcomes are still needed. There is still a lack of randomized control trials (RCTs) assessing the efficacy and safety of energy based devices for vaginal "rejuvenation." Many unanswered questions still remain for example the long term impact on tissues and on adjacent nerves, vessels, bladder, rectum, etc is unknown. The unregulated use of vaginal laser will make it difficult to assess any long-term impact. There may be an effect on surgical dissection and treatment outcomes in the future if women eventually seek appropriate reconstructive pelvic or anti-incontinence surgery.

Ideally any future RCTs should compare treatment with a sham treatment to assess the impact of the placebo effect. To provide context, data has shown that $70 \%$ of the improvement outcome for women undergoing treatment for sexual dysfunction, is the result of placebo treatment. ${ }^{16}$ It is therefore expected that women seeking vaginal rejuvenation for sexual dysfunction would find improvement, even in the absence of a biological effect.

Vaginal laser therapy has also been used for defined gynaecological conditions, for example, treatment of vulvovaginal atrophy. However assertions for vaginal rejuvenation by laser is not sustained by histological evidence or long term studies and is misleading. Women can alternatively be effectively and safely treated with topical oestrogen even in women with previous cancer or are unable to use systemic hormone replacement therapy. ${ }^{17,18}$ Similarly, claims that vaginal laser is a treatment option for women with vulvodynia and lichen sclerosus is again not supported by scientific evidence, lacks biological plausibility, and can cause potential harm. The appeal of vaginal laser treatment as an office procedure for female pelvic organ prolapse, the so called "vaginal relaxation syndrome" and stress urinary incontinence has also been explored. ${ }^{19-29}$ There is minimal published data with the recurring theme of paucity of good quality evidence in the form of multi-center randomized placebo-controlled trials, which is concerning.

If treatment with laser/energy devices are attractive to both the woman and clinician, in the absence of strong 
scientific evidence on the clinical role of laser treatment, appropriate pretreatment counseling addressing potential and known risks and benefits is mandatory. Established treatment alternatives should also be offered. We strongly believe that physicians have an ethical responsibility to provide their patients with accurate information regarding the efficacy and safety of innovative practices, especially when using/ promoting new devices for treatments whose efficacy and safety are still not completely known. ${ }^{17}$

In the informed decision-making process relational factors should be considered, as sexuality in postmenopausal women is a complex mosaic of physical, psychological, socioeconomic, and cultural factors. An approach simply focused to the "vaginal mucosa" is limited and insufficient. ${ }^{5}$

\section{2 | CONCLUSIONS}

The FDA has not yet determined whether it is safe or practical to use energy-based devices such as lasers for "rejuvenation" procedures or the aforementioned described symptoms and gynaecological conditions. ${ }^{1,2,5}$ Until further robust scientific data emerges, this technology should be considered experimental.

We believe that the use of laser and radiofrequency energy based devices for the treatment of lichen sclerosus, vulvodynia, urinary incontinence, sexual dysfunction, pelvic organ prolapse, urinary tract infections, vulvovaginal atrophy, or for vaginal "rejuvenation," should only be used within the research setting of properly designed clinical trials. Patients enrolled in these studies should be appropriately and clearly counseled, consented and followed up. The follow-up protocol should be sufficiently long enough to establish the long-term effects of vaginal laser and radiofrequency. It remains unknown if any negative impact may become evident several months or years after treatment or following repeated treatments.

If patients are determined to undergo vaginal "rejuvenation" as a therapy outside of a trial, it is the clinician's ethical responsibility to inform patients about the lack of data and uncertainty on efficacy and safety of the therapy and provide known information about potential complications. Arrangements for clinical governance, consent, and audit should be made.

\section{ORCID}

Giuseppe Alessandro Digesu (iD http://orcid.org/0000-00023914-8147

\section{REFERENCES}

1. U.S. Food and Drug Administration. 510(k) Summary for RevLite Q-Switched Nd:YAG Laser System [letter]. Silver Spring (MD): FDA; 2014. Available at: http://www.accessdata.fda.gov/ cdrh_docs/pdf13/k133254.pdf. Retrieved May 19, 2016.
2. Committee on Gynecologic Practice, American College of Obstetricians and Gynecologists. ACOG committee opinion No.378: "Vaginal rejuvenation" and cosmetic vaginal procedures. Obstet Gynecol 2007;110:737-738.

3. FDA Warns Against Use of Energy-Based Devices to Perform Vaginal "Rejuvenation" or Vaginal Cosmetic Procedures: FDA Safety Communication. https://www.fda.gov/MedicalDevices/ Safety/AlertsandNotices/ucm615013.htm.

4. Streicher LF. Vulvar and vaginal fractional $\mathrm{CO} 2$ laser treatments for genitourinary syndrome of menopause. Menopause. 2018;25: $571-573$

5. U.S. Food and Drug Administration. Re: trade/device name: DEKA SmartXide2 Laser System [letter]. Silver Spring (MD): FDA; 2014. Available at: http://www.accessdata.fda.gov/cdrh_docs/pdf13/ k133895.pdf. Retrieved May 19, 2016.

6. Vieira-Baptista P, Damaser M, Digesu A, Marchitelli C, Preti M, Stockdale C. To the Editor. Menopause. July 2018 1. https://doi.org/ 10.1097/GME. 0000000000001165.

7. Fractional laser treatment of vulvovaginal atrophy and U.S. Food and Drug Administration Clearance. https://www.acog.org/ Clinical-Guidance-and-Publications/Position-Statements/ Fractional-Laser-Treatment-of-Vulvovaginal-Atrophy-and-USFood-and-Drug-Administration-Clearance.

8. Fractional microablative $\mathrm{CO} 2$ laser in breast cancer survivors affected by iatrogenic vulvovaginal atrophy after failure of nonestrogenic local treatments: a retrospective study: erratum. Menopause. 2018;25:1169.

9. Digesu GA, Swift S. Laser treatment in urogynaecology and the myth of the scientific evidence. Int Urogynecol J. 2017;28: 1443-1444.

10. Singh A, Swift S, Khullar V, Digesu GA. Laser vaginal rejuvenation: not ready for prime time. Int Urogynecol J. 2015; 26:163-164.

11. ACOG committee opinion No. 378: vaginal "rejuvenation" and cosmetic vaginal procedures. Obstet Gynecol. 2007;110:737-738.

12. Walter J-E, Larochelle A. No. 358-Intravaginal laser for genitourinary syndrome of menopause and stress urinary incontinence. J Obstet Gynaecol Canada. 2018;40:503-511.

13. Vieira-Baptista P, Almeida G, Bogliatto F, et al. International society for the study of vulvovaginal disease recommendations regarding female cosmetic genital surgery. J Low Genit Tract Dis 2018;22:415-434.

14. Cruz VL, Steiner ML, Pompei LM, et al. Randomized, doubleblind, placebo-controlled clinical trial for evaluating the efficacy of fractional $\mathrm{CO} 2$ laser compared with topical estriol in the treatment of vaginal atrophy in postmenopausal women. Menopause. 2018; 25:21-28.

15. Statement from FDA Commissioner Scott Gottlieb, M.D., on efforts to safeguard women's health from deceptive health claims and significant risks related to devices marketed for use in medical procedures for "vaginal rejuvenation". https://www. fda.gov/newsevents/newsroom/pressannouncements/ucm615130. htm

16. Weinberger JM, Houman J, Caron AT, et al. Female Sexual Dysfunction and the Placebo Effect. Obstet Gynecol. 2018;132: 453-458.

17. ACOG committee on ethics. ACOG committee opinion No. 352: innovative practice: ethical guidelines. Obstet Gynecol. 2006;108: 1589-1595. 
18. Rueda C, Osorio AM, Avellaneda AC, Pinzón CE, Restrepo OI. The efficacy and safety of estriol to treat vulvovaginal atrophy in postmenopausal women: a systematic literature review. Climacteric. 2017;20:321-330.

19. Gaspar A, Addamo G, Brandi H. Vaginal fractional CO 2 laser: a minimally invasive option for vaginal rejuvenation. Am J Cosmet Surg. 2011;28:156-162.

20. Ogrinc UB, Senčar S, Lenasi H. Novel minimally invasive laser treatment of urinary incontinence in women. Lasers Surg Med. 2015;47:689-697.

21. Fistonić N, Fistonić I, Guštek ŠF, et al. Minimally invasive, non-ablative Er:YAG laser treatment of stress urinary incontinence in women-a pilot study. Lasers Med Sci. 2016;31:635-643.

22. Fistonić N, Fistonić I, Lukanovič A, Findri G, Sorta Bilajac Turina I, Franić D. First assessment of short-term efficacy of Er:YAG laser treatment on stress urinary incontinence in women: Prospective cohort study. Climacteric. 2015;18:37-42.

23. Pardo JI, Solà VR, Morales AA. Treatment of female stress urinary incontinence with Erbium-YAG laser in non-ablative mode. Eur J Obstet Gynecol Reprod Biol. 2016;204:1-4.

24. Gaspar A, Brandi H. Non-ablative erbium YAG laser for the treatment of type III stress urinary incontinence (intrinsic sphincter deficiency). Lasers Med Sci. 2017;32:685-691.

25. Gambacciani M, Torelli MG, Martella L, et al. Rationale and design for the vaginal erbium laser academy study (Velas): An international multicenter observational study on genitourinary syndrome of menopause and stress urinary incontinence. Climacteric. 2015;18:43-48.

26. Gaviria JE, Lanz JA. Laser vaginal tightening (LVT)—evaluation of a novel noninvasive laser treatment for vaginal relaxation syndrome. J Laser Heal Acad Artic J LAHA. 2012;1:59-66.

27. Bizjak Ogrinc U, Sencar S, Non-surgical minimally invasive Er: YAG laser treatment for higher-grade cystocele. NDP 373, 38th Annual IUGA Meeting, Dublin, Ireland; May 28th-June 1st, 2013.

28. Bizjak-Ogrinc U, Sencar S, Vizintin Z. \#178 3 years follow-up of pelvic organ prolapse treated with Er:YAG laser. 37th American Society for Laser Medicine and Surgery, San Diego, USA 5-9 April 2017.

29. da Silva Lara LA, Useche B, Rosa e Silva JC, et al. Sexuality during the climacteric period. Maturitas. 2009;62:127-133.

How to cite this article: Digesu GA, Tailor V, Preti $\mathrm{M}$, et al. The energy based devices for vaginal "rejuvenation," urinary incontinence, vaginal cosmetic procedures, and other vulvo-vaginal disorders: An international multidisciplinary expert panel opinion. Neurourology and Urodynamics. 2019;1-4. https://doi.org/10.1002/nau.23927 